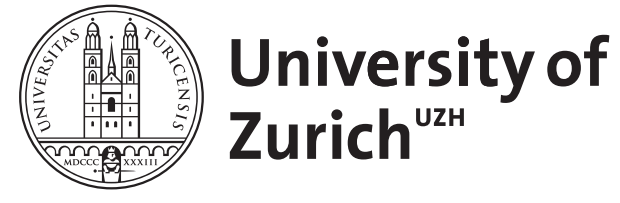

Zurich Open Repository and Archive

University of Zurich

University Library

Strickhofstrasse 39

CH-8057 Zurich

www.zora.uzh.ch

Year: 1994

\title{
Neue Konzepte zum Abbau der Arbeitslosigkeit
}

Ruh, Hans

DOI: https://doi.org/10.14315/zee-1994-0103

Posted at the Zurich Open Repository and Archive, University of Zurich

ZORA URL: https://doi.org/10.5167/uzh-154031

Journal Article

Published Version

Originally published at:

Ruh, Hans (1994). Neue Konzepte zum Abbau der Arbeitslosigkeit. Zeitschrift für evangelische Ethik, 38(1):2-3.

DOI: https://doi.org/10.14315/zee-1994-0103 


\section{Kommentar}

\section{Neue Konzepte zum Abbau der Arbeitslosigkeit}

\section{Von Hans Ruh}

Arbeitslosigkeit ist zweifellos ein schwerwiegendes Problem auf der ganzen Welt. Erstaunlich sind aber, je länger desto mehr, zwei Dinge: Einmal, daß es die westliche Industriegesellschaft fertigbringt, mit der Arbeitslosigkeit leidlich zu leben, und zweitens, daß uns zur Lösung dieses Problems eigentlich nichts einfällt. Beides kann, aller Voraussicht nach, nicht so weitergehen. Denn es ist eben doch undenkbar, daß die tiefgreifenden sozialen, ökonomischen und sozialpsychologischen Probleme der Arbeitslosigkeit auf die Dauer ohne schwerwiegende Konflikte bleiben werden. Erstaunlich ist aber, daß zur Lösung dieser Probleme nur Ladenhüter angeboten werden: Eingriffe in die Wirtschaft im Sinne von Keynes, Arbeitsbeschaffungsprogramme durch den Staat, Teilung der vorhandenen Arbeit, mäßige Arbeitszeitsenkung. Dabei wäre es wohl an der Zeit, das Problem der Arbeitslosigkeit auf die Ebene zu legen, auf die es gehört. Es handelt sich dabei um eine tiefgreifende Krise des menschlichen Tätigkeitshaushalts.

Anzeichen dafür sind Widersprüchlichkeiten in der Arbeitsgesellschaft, z. B. diese: Immer weniger Menschen arbeiten immer mehr, immer mehr Menschen immer weniger. Wir haben viele ungelöste Probleme: Umwelt, Hunger, Soziales, Wohnen, und doch bringen wir es nicht zustande, die menschliche Tätigkeit auf diese Probleme anzusetzen. Oder: Die rationalisierte Arbeit gefährdet immer mehr die Umwelt. Oder: Neue Rationalisierungswellen wie die der Lean-Production filtern immer mehr nicht angepaßte Menschen aus dem Arbeitsprozeß heraus. Oder: Wir produzieren Produkte, die wir eigentlich nicht brauchen. Die Gründe für eine solchermaßen zu beschreibende Krise des menschlichen Tätigkeitshaushalts sind mannigfach. Im Zentrum ist sie wohl die Abkoppelung der Tätigkeitssteuerung von den vitalen Bedürfnissen.

Aber nun zu der wichtigsten Frage: Welchen Beitrag kann die theologische Ethik zur Lösung dieser Krise leisten? Ohne Zweifel gibt es eine imposante Geschichte der theologischethischen Deutung der Arbeit von der Bibel bis zur Gegenwart. Karl Barth hat in der kirchlichen Dogmatik (III,4) zentrale Gesichtspunkte festgehalten, etwa: Arbeit hat Dienstfunktion, z. B. am Nächsten, aber auch an der Schöpfung Gottes. Arbeit muß sich auf sinnvolle Zwecke ausrichten. Arbeit ist immer humane Arbeit, z. B. im Blick auf Arbeitsbedingungen und Konkurrenz. Arbeit soll dazu dienen, daß wir anderen Menschen nicht zur Last fallen. Arbeit dient der Deckung fundamentaler Bedürfnisse, ist aber zugleich der Ort menschlicher Entfaltung.

Es leuchtet ein, daß diese idealen Zielsetzungen für die menschliche Arbeitswelt ein Rezept zur Lösung der Krise sein könnten, wenn sie die Chance bekämen, sich mit der harten 
Wirklichkeit zu vermitteln. Denn diese Zielsetzungen sind das Produkt von positiven Erfahrungen darüber, wie der menschliche Tätigkeitshaushalt gelingen kann.

Es könnte sich nun die Situation ergeben, daß der Leidensdruck der Arbeitslosigkeit so gro $\beta$ wird, daß die Suche nach neuen Lösungen ultimativ wird. Dann würden die genannten theologischen Zielsetzungen der Arbeit attraktiv. Aber wo liegt die Chance ihrer praktischen Umsetzung?

Diese Umsetzung wird nicht möglich sein, ohne daß wir zwei fundamentale Veränderungen ins Auge fassen. Die erste Veränderung betrifft eine teilweise Entkoppelung von Arbeit und Lohn. Die zweite besteht in der Unterordnung der monetarisierten Arbeitszeit unter den Oberbegriff einer Tätigkeitsgesellschaft mit einer Vielzahl von Tätigkeitszeiten.

Auf diese Weise ließe sich zunächst die fatale Dynamik der Rationalisierung der Arbeit mit deren negativen Folgen brechen. Die Entkoppelung von Arbeit und Lohn, d. h. die Auszahlung eines Grundlohns oder einer Bürgerrente für alle, brächte die ökonomische Voraussetzung dafür, daß Menschen neben der monetarisierten Arbeitszeit anderen, selbstgewählten, ökologisch und sozial sinnvollen Tätigkeiten nachgehen können. Schematisch sind folgende Weisen nichtmonetarisierter Tätigkeiten vorstellbar: Freizeit, Sozialzeit, und zwar teilweise im Sinne eines obligatorischen Sozialdienstes, Eigenarbeitszeit, Ichzeit und Reproduktionszeit, d. h. die Zeit, welche der kommenden Generation, also den Kindern, gewidmet wird.

Die entscheidenden Vorteile einer solchen Konzeption sind: Die Förderung einer freiwilligen und obligatorischen Ausrichtung der menschlichen Tätigkeit auf sozialpsychologisch, sozial und ökologisch wichtige Ziele, deren Erreichung personalintensiv ist oder bisher nicht finanzierbar war. Die Einordnung aller in einen quantitativ reduzierten Arbeitsprozeß, in dem die 50\%-Stelle zur (freiwilligen) Norm werden könnte. Die Senkung von Gesundheits-, Gewalt- und Umweltkosten infolge der stärkeren Zuwendung zu anderen Menschen und zu sich selbst. Natürlich ist eine solche Neuordnung nicht ohne Probleme, z. B. im Blick auf die Attraktivität eines solchen Modells für die ökonomisch bedingten Wanderungsbewegungen. Es stellen sich auch Probleme der Konkurrenzfähigkeit. Aber fairerweise sollte man ein solches Modell immer messen an den negativen Fakten der heute geltenden Ordnung. Wir haben gelernt, mit schwerwiegenden Problemen umzugehen, indem wir sie verdrängen, etwa die Kosten der Umweltzerstörung, die Kosten der Gewalt, die Lage der alten Menschen, die sozialen Folgen der Arbeitslosigkeit.

Und wenn die heutige Arbeitswelt das Produkt tiefgreifenden Wandels darstellt: Warum soll man nicht auch das Verhältnis von Lohn und Arbeit durch die Aufteilung der Tätigkeitszeit wandeln können?

Prof. Dr. Hans Ruh

Institut Sozialethik an

der Universität Zürich

Kirchgasse 9

CH-8001 Zürich 